\title{
TRABAJANDO LA INCONTINENCIA URINARIA EN ATENCIÓN PRIMARIA: SATISFACCIÓN, SEXUALIDAD Y CUMPLIMIENTO TERAPÉUTICO.
}

\author{
Luis García-Giralda Ruíz', Lorenzo Guirao Sánchez², Isabel Casas Arandal, Juan Vicente Alfaro \\ González², Gregorio Sánchez Pérez³ y Lorenzo Guirao Egea 4 .
}

'Centro de Salud Murcia-Centro, ${ }^{2}$ Centro de Salud de Alguazas, ${ }^{3}$ Consultorio Simón García. ${ }^{4}$ Facultad de Medicina de Murcia. Gerencia de Atención Primaria de Murcia. SMS. Murcia. España.

\begin{abstract}
Resumen.- OBJETIVO: Pretendemos valorar la satisfacción de los pacientes de ambos sexos con incontinencia urinaria y secundariamente pretendemos analizar la influencia de la incontinencia de urgencia en la sexualidad de nuestros pacientes y el cumplimiento terapéutico en aquellos pacientes con incontinencia de urgencia.
\end{abstract}

MÉTODO: Se ha realizado un estudio naturalístico quasi-experimental, longitudinal, abierto, no controlado, durante 24 semanas con análisis antes-después, de 60 pacientes diagnosticados de incontinencia urinaria de urgencia o mixta, y tratados con terapia física y tolterodina, utilizando el cuestionario sobre vejiga hiperactiva $O A B-q$, versión española, el cuestionario de calidad de vida King's Health Questionnaire (KHQ) versión española, el de satisfacción sexual Golombok Rust Inventory of Sexual Satisfaction (G.R.I.S.S.I y valorando el cumplimiento terapéutico.
RESULTADOS: De los calendarios valorables, el 56,25\% (18) presentan al finalizar el estudio criterios de "normalidad" miccional $(p<0,001)$. Se ha producido una mejoría de todos los ítems del OAB-p tras la intervención del 26,55\% (p<0,001). También se ha producido una mejora significativa $(p<0,001)$ en la percepción del estado de salud en el $K H Q$, pero no ha sucedido lo mismo con el GRISS. Finalmente se puede constatar una mejora en los diversos items sobre cumplimiento terapéutico.

CONCLUSIONES: Los resultados de este estudio vienen a corroborar la eficacia conjunta de medidas de rehabilitación de suelo pélvico y terapia farmacológica en el tratamiento de la incontinencia urinaria. Además son muy esperanzadores, en el sentido de que el problema de incontinencia está influyendo negativamente en su percepción de salud, y en la medida en que intervengamos sobre la incontinencia de estos pacientes mejoraremos su "estado de salud

Palabras clave: Incontinencia urinaria. Calidad de vida. Sexualidad. Cumplimiento terapéutico.

Summary.- OBJECTIVES: Our aim is not only to measure the satisfaction of both sex patients with urinary incontinence but also to analyse the influence of urgency incontinence in our patient's sexuality as well as their treatment fulfilment.

METHODS: This is a quasi-experimental naturalistic, longitudinal, open and not controlled study made during 24 weeks with before-after analysis on 60 urgent or mixed urinary incontinence patients treated with physic therapy and tolterodine. We have used the Spanish version of the questionnaire about overactive bladder (OAB-q), the Spanish version of the King's Health Questionnaire (KHQ) and the Golombok Rust Inventory of Sexual Satis- 
faction (G.R.I.S.S.) about sexual satisfaction. We have born in mind treatment fulfilment.

RESULTS: The 56,25\% (18) of the valid calendars showed "normal" mictional values at the end of the study $(p<0,001)$. Each item of the OAB-q has improved after the treatment of the 26,55\% $(p<0,001)$. The health status has also improved in the $K H Q(p<0,001)$, but it hasn't happened the same in the G.R.I.S.S. Finally, an improvement in several items of the treatment fulfilment can be contrasted.

CONCLUSIONS: The results of this study corroborate the efficiency of pelvic floor rehabilitation combined with pharmacological therapy in urinary incontinence treatment. These results are very hope-giving because the incontinence problem affects negatively to the patients' perception of their own health status, so solving this problem their health status will be improved.

Keywords: Urinary incontinence. Quality of life. Sexuality. Treatment fulfilling.

\section{INTRODUCCIÓN}

La incontinencia urinaria ha sido definida como un síntoma o condición, consistente en la pérdida involuntaria de orina demostrable de forma objetiva y que constituye un problema social y familiar para la persona que lo sufre, reflejando en la actualidad un problema importante de salud en sus aspectos médicos, sociales y económicos, afectando a la calidad de vida de las personas que la padecen. La prevalencia de la incontinencia es difícil de precisar variando considerablemente en función de los estudios epidemiológicos de un $2 \%$ a un $55 \%$, debido fundamentalmente a la diferencia de criterios utilizados. En general afecta más a las mujeres que a los hombres, sobre todo a postmenopausicas (1), aumentando su prevalencia con la edad, siendo los pacientes ancianos los que más la padecen (40\%) (2). La incontinencia de esfuerzo es la más frecuente en mujeres jóvenes y al aumentar la edad se incrementa la incontinencia de urgencia, y la incontinencia mixta pasa a ser el tipo más frecuente en las personas mayores (3).

En mujeres aumenta la incidencia con la edad, número de hijos y la menopausia (4). En varones suele tener relación con patología prostática. En ambos sexos suele verse influenciada por la presencia de diabetes, patología neurológica (ACV, Parkinson, demencia, hidrocefalia y tumores), intervenciones en zona pélvica o abdominal, utilización de algún tipo de fármaco (medicaciones neuropsiquiátricas, betaestimulantes, bloqueadores alfa), fecalomas, imposibilidad de movilización oportuna y radioterapia (5).
La Calidad de Vida Relacionada con la Salud (CVRS), entendida como un resumen de características relacionadas con el bienestar y la capacidad de actuación de las personas afectadas por determinado problema de salud, supone la capacidad del individuo para lograr sus objetivos y llevar a cabo su "proyecto vital" en un mundo significativo. El bienestar y la capacidad de actuación son fenómenos, ante todo, valorables por el propio enfermo que es quien los vive y no para quien desde fuera los contempla (en este caso los profesionales sanitarios) (6). Esto convierte a la medición del estado de salud o de la percepción de la calidad de vida en una novedosa tecnología comunicativa que toma en cuenta el punto de vista del paciente en el proceso terapéutico, lo que ha llevado al Comité Científico Internacional (Paris 2002) (7) a establecer entre las pruebas diagnósticas recomendables "la valoración de la calidad de vida y síntomas adicionales".

La incontinencia urinaria tiene un efecto notable sobre la calidad de vida de los individuos afectados, de forma que el $60 \%$ se sienten avergonzados o preocupados por sus síntomas. Un $60 \%$ de las mujeres afectadas evitan alejarse de sus hogares, un $45 \%$ no utiliza transporte público y $50 \%$ rechaza la actividad sexual, teniendo mayor predisposición a problemas emocionales y a aislamiento social (8). En este sentido, los consensos internacionales como el mencionado de Paris 2002, establecen como pruebas altamente recomendables "la valoración del impacto de la incontinencia en la sexualidad" y de "los aspectos social y cultural" que rodean a estos pacientes. A pesar del problema que reflejan estos datos, sólo el $6 \%$ de los individuos con incontinencia moderada o grave reciben algún tipo de asesoramiento y asistencia médica, al ser una patología mal conocida y escasamente abordada desde atención primaria, interrogando poco sobre ella, no llegando a un diagnóstico etiológico y ofreciéndose a veces sólo medidas paliativas como el uso de pañales (9).

Hasta un $50 \%$ de los pacientes no consultan al médico a pesar de experimentar la incontinencia como un problema. Las razones por las que no buscan atención médica son variadas. Unos pueden aceptar su situación y aprender a vivir con su sufrimiento formando parte de su vida cotidiana, pero otra gran parte de estos pacientes creen que es un problema sin tratamiento o que existe un conocimiento poco preciso del mismo por parte del personal médico (10). En otras ocasiones son los mismos profesionales sanitarios los que mostramos una falta de sensibilidad, minimizando la importancia e implicaciones de la incontinencia urinaria, no prestándoles la suficiente atención a estos pacientes. 
Por otra parte, aunque tenemos tratamientos farmacológicos específicos y eficaces, como los antimuscarínicos, para la incontinencia de urgencia, el cumplimiento terapéutico a medio y largo plazo es bastante bajo a la luz de nuestra experiencia. Es precisamente este uno de los motivos principales que ha conducido a nuestro Equipo de Atención Primaria a realizar desde enero de 2002 un abordaje específico para aflorar esta patología oculta en los pacientes atendidos en nuestros Centros de Salud. En un estudio previo realizado en Atención Primaria durante 20022003 (11), fue necesario incluir a 90 pacientes para conseguir que finalizaran 52 de ellos, debido a:

1). 21 pacientes $(55,26 \%)$ por cumplimiento terapéutico inferior al $80 \%$ de la dosis prescrita,

2). el 15,79\% (6 pacientes) por anulación de los cuestionarios (respuestas múltiples o falta de respuesta), $y$

3). 11 por abandono del estudio $(28,95 \%), 5$ de ellos por efectos secundarios.

La evaluación del desarrollo de este programa y las conclusiones de la mencionada revisión es lo que nos orienta para seguir trabajando sobre cuál debería ser el abordaje más adecuado de nuestros pacientes con incontinencia urinaria.

\section{OBJETIVOS}

El objetivo principal es valorar la satisfacción, en términos de calidad de vida, de los pacientes de ambos sexos con incontinencia urinaria tratados en consulta de atención primaria.

Secundariamente pretendemos analizar la influencia de la incontinencia de urgencia en la sexualidad de nuestros pacientes y el cumplimiento terapéutico en aquellos pacientes con incontinencia de urgencia.

\section{MÉTODOS}

Se ha realizado un estudio naturalístico quasi-experimental, longitudinal, abierto, no controlado, durante 24 semanas con análisis antes-después, de pacientes diagnosticados de incontinencia urinaria siguiendo las recomendaciones de Paris 2002 del Comité Científico Internacional para la evaluación y tratamiento de la incontinencia urinaria (7), valorando su calidad de vida, satisfacción sexual y cumplimiento terapéutico.

Los pacientes que han expresado su consentimiento a participar en el estudio, se han incluido de forma consecutiva hasta el total de la muestra inicialmente establecida de 50 pacientes $(\alpha=0.05$, $\beta=0.20$ ), que incrementamos hasta 60 para controlar los posibles abandonos. Como criterios de inclusión: ser mayor de 18 años, presentar algún grado incontinencia de urgencia o mixta y que hayan aceptado participar en el estudio. Los criterios de exclusión han sido: ser menores de 18 años o presentar incontinencia de esfuerzo.

Las variables evaluadas figuran en la Tabla I.

La intervención ha consistido en tratamiento rehabilitador de la incontinencia mediante ejercicios de kegel (12) y farmacológico con $4 \mathrm{mg}$ una vez al día de tolterodina extended-release, recibiendo información detallada sobre el problema que le afecta y sobre la importancia de seguir las recomendaciones sobre estilo de vida y la toma de la medicación conforme a la prescripción. El motivo de elegir este fármaco ha sido, entre otros, su mejor relación costeefectividad y tolerancia, como reflejan las publicaciones recientes en este sentido (13-16).

\section{RESULTADOS}

\section{Datos generales}

Se analizan los datos referentes a 60 pacientes seguidos durante 24 semanas en el periodo comprendido desde la autorización del estudio por la Gerencia de Atención Primaria de Murcia el 15 de marzo de 2004 a junio de 2005.

Desde su inicio se ha producido un elevado porcentaje de "rechazo", aproximadamente 1 de cada 3 pacientes (32) invitados a participar en el estudio $(34,78 \%)$, en donde no se ha obtenido el consentimiento en la primera visita, una vez realizados los test para valorar su incontinencia urinaria, calidad de vida y fundamentalmente la satisfacción sexual. Además se han producido 9 abandonos por perdida de seguimiento.

La edad media es de 65,21 años, con sobrepeso (IMC de 27,89$)$ y de sexo mayoritariamente femenino $(71.66 \%)$.

En cuanto a patologías concomitantes, el $54,92 \%$ tienen artrosis, un $25,49 \%$ de los pacientes presenta diabetes mellitus tipo 2, un 45,09\% HTA, el $15,68 \%$ patología cardiovascular y el $41,17 \%$ síndrome ansioso-depresivo; el $75 \%$ de los varones presentaban patología prostática.

El calendario miccional sólo lo han entregado al finalizar el estudio 32 pacientes $(62,74 \%)$. El resto manifiesta haberlo llevado asiduamente pero por diversos motivos no lo tienen en la visita final. De los 
calendarios valorables, el 56,25\% (18) presentan al finalizar el estudio criterios de "normalidad" miccional (menos de 8 al día y/o menos de 2 por la noche) (d 26,35, t-Student -9.012, $p<0,001$ ) (Figura 1).

Valoración del impacto de la incontinencia: cuestionario sobre vejiga hiperactiva $O A B-q$, versión española.

Se trata de un cuestionario auto-administrado con 33 preguntas con respuesta múltiple, distribuidas en 2 bloques. El primero de 8 preguntas sobre las molestias de los síntomas de incontinencia, y el segundo de 25 sobre el impacto que esos síntomas han tenido en su vida.

La puntuación promedio inicial para los 33 ítem del cuestionario fue de 3,42 (dt 1.032) y la puntuación media final ha sido de 2,51 (dt 0,618). Las diferencias observadas en el incremento medio de todos los ítems han sido estadísticamente significativas con una mejora relativa tras la intervención del 26,55\% (t-Student $-4,34, p<0,001$, con corrección de Welch para varianzas no homogéneas). En cambio, no ha habido variación significativa para los ítems 12 (los síntomas, le causaron angustia?), 13 (le hicieron sentirse frustrado/a?), 18 (hicieron que sus amigos y familia se sintiera frustrados?) y 27 (influyeron en sus relaciones con familiares y amigos?) (Figura 2).

\section{Calidad de vida: KHQ (King's Health Questionnaire. versión española)}

Con 21 preguntas distribuidas en bloques: estado de salud, actividades diarias, limitaciones físicas y sociales, relaciones personales, emociones, sueño/vitalidad e higiene personal.

Se observaron diferencias estadísticamente significativas, al comparar la visita final frente a la visita basal, utilizando el test no paramétrico de Wilcoxon para muestras relacionadas.

En la componente "estado de salud" del cuestionario se observaron diferencias significativas entre la puntuación media inicial y la puntuación media final del grupo intervención (d 1.88; IC 95\%; 1.34$2.26 ;+3.264 ; p<0.01)$. Algo similar ha ocurrido en

TABLA I. ESQUEMA GENERAL DEL ESTUDIO.

\begin{tabular}{|l|c|c|c|c|}
\hline \multicolumn{1}{|c|}{ Visitas } & Basal & 4 semana & 12 semana & 24 semana \\
\hline Datos sociodemográficos & $X$ & & & \\
\hline Historia clínica & $X$ & & & \\
\hline Exploración física & $X$ & & & $X$ \\
\hline Diario miccional & $X$ & $X$ & $X$ & $X$ \\
\hline Analítica y pruebas complementarias & $X$ & & & $X$ \\
\hline Patologías concomitantes & $X$ & $X$ & $X$ & $X$ \\
\hline Cuestionario OAB-q de incontinencia urinaria & $X$ & & & $X$ \\
\hline Cuestionario GRISS sobre satisfacción sexual & $X$ & & & $X$ \\
\hline Cuestionario de Calidad de Vida KHQ & $X$ & & & $X$ \\
\hline Cumplimiento terapéutico (PC) & & & $X$ & $X$ \\
Recuento de pastillas/envases prescritos & & $X$ & $X$ & $X$ \\
Cumplimiento autocomunicado (CA) & & $X$ & $X$ & $X$ \\
Cumplimiento percibido por el paciente (EVA) & & $X$ & $X$ & $X$ \\
Test de Morisky-Green & & $X$ & $X$ & $X$ \\
\hline Intervención: & & $X$ & $X$ & $X$ \\
Rehabilitación, tolterodina y educación sanitaria & & & & $X$ \\
\hline Tolerancia y efectos adversos & & $X$ & $X$ & $X$ \\
\hline
\end{tabular}


la componente "actividades diarias" donde se observaron diferencias significativas entre la puntuación media inicial y la puntuación media final (d 1.03; IC 95\%; 0.73-1.84; † 2.783; $\mathrm{p}<0.05)$. En "limitaciones físicas y sociales" se observaron diferencias significativas entre la media inicial y final (d 3.88; IC 95\%; $3.01-4.76 ; \uparrow 4.264 ; p<0.01)$. En el bloque sobre "relaciones personales" las diferencias entre las visitas inicial y final han sido escasas (d 0.95 IC $95 \%$; 0.39 $1.81 ;+2.813$; $\mathrm{p}<0.05)$, lo mismo que con la componente "emociones" (d 0.75 IC 95\%; 0.66-1.21; † 2.643; $\mathrm{p}<0.05$ ), y la de "sueño/vitalidad" (d 0.86 IC 95\%; 0.43-1.72; † 1.972; $\mathrm{p}<0.05)$. Finalmente, en el bloque de "higiene personal" es donde se ha producido uno de los cambios más intensos (d 3.32; IC 95\%; 2.10-4.54; † 3.764; p<0.001) (Figura 3).

\section{Cuestionario de satisfacción sexual G.R.I.S.S. (Golom- bok Rust Inventory of Sexual Satisfaction)}

Se trata de un cuestionario auto-administrado de 31 preguntas con respuesta múltiple.

No se han observado diferencias significativas en las puntuaciones medias entre la visita inicial y final $(73,71$ y 72,85 respectivamente), excepto para la ítem 20 " ¿rechaza tener relaciones sexuales con su pareja?" que ha mejorado significativamente respecto a la visita inicial (d 0,55, t-Student $-3,56$, $p<0,001$ ), lo mismo que para el ítem 30 " ¿tiene escapes de orina durante el acto sexual?" (d 0,45, t-Student $-2,49, \mathrm{p}<0,01)$.

\section{Cumplimiento terapéutico (17)}

\section{- Cumplimiento Autocomunicado (CA)}

Se observaron diferencias estadísticamente significativas en la pregunta "la mayoría de las personas por una razón u otra, tienen dificultad en

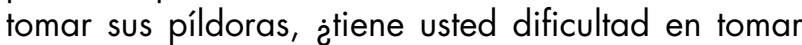
las suyas?", al comparar la visita final frente a la vi- sita a la $4^{\underline{a}}$ semana, utilizando el test no paramétrico de Wilcoxon para muestras relacionadas (Z -3.024; $\mathrm{p}<0.05)$.

\section{- Cumplimiento Objetivo (PC) (Figura 4)}

En el grupo estudiado se observaron diferencias estadísticamente significativas al preguntar " $\dot{\mathrm{H}} \mathrm{Ha}$ traído los envases?", al comparar la visita final (semana 24) frente a la visita a las 4 semanas, utilizando el test no paramétrico de Wilcoxon para muestras relacionadas ( $Z-2.828 ; p<0.05)$. No ocurre lo mismo cuando comparamos la visita de las 4 semanas con la de 12.

PC HTA ( $n^{\circ}$ comprimidos tomados/ $n^{\circ}$ comprimidos prescritos $x$ 100)

Se observaron diferencias estadísticamente significativas entre la puntuación total media en la visita a las 4 semanas y la puntuación total media final (d 15.31; IC 95\%; 10.55-20.06; † 6.502; p<0.001). También se produjeron diferencias significativas pero de menor intensidad al comparar la visita a las 4 semanas con de 12 (d 2.94; IC 95\%; 1.96-3.91; † 6.064; $p<0.001)$.

\section{- Test de Moriski-Green}

Se observaron diferencias estadísticamente significativas, al comparar la visita final frente a la visita a las 4 semanas, utilizando el test no paramétrico de Wilcoxon para muestras relacionadas de las siguientes preguntas relacionadas con el consumo de tolterodina:

1.- ¿En alguna ocasión ha olvidado tomarse la medicación?, en el grupo en estudio ( $Z$-5.096; $\mathrm{p}<0.001)$.

2.- ¿Le ha sido posible tomarse el fármaco a las horas indicadas por el médico? Se han observado diferencias significativas para el grupo (Z -4.000; $\mathrm{p}<0.001)$.

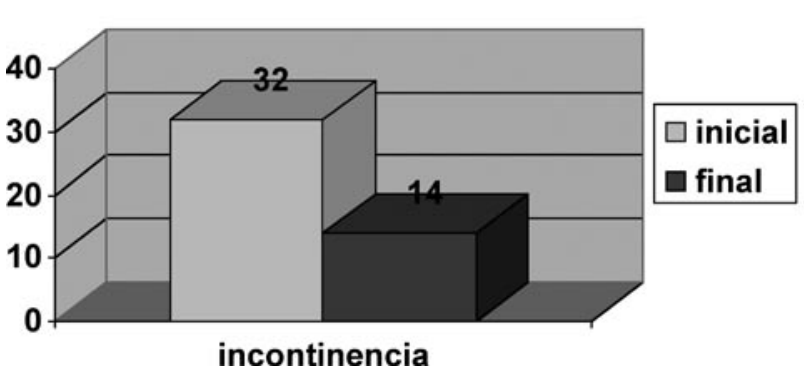

FIGURA 1. Pacientes con incontinencia según diario miccional.

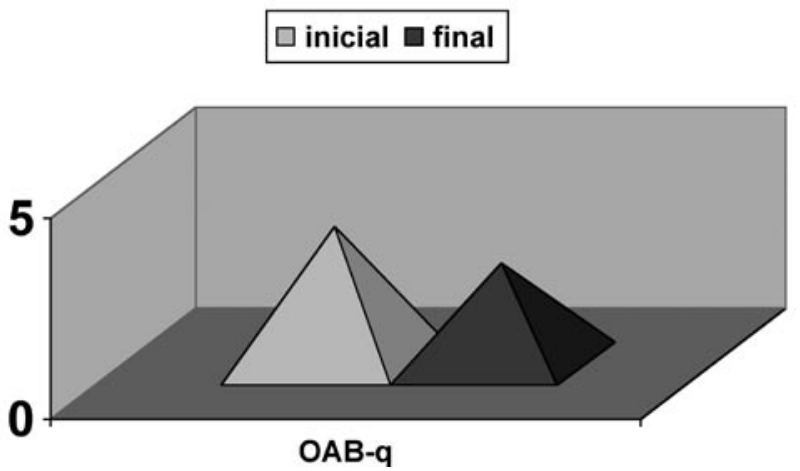

FIGURA 2. Disminución de síntomas de incontinencia. 


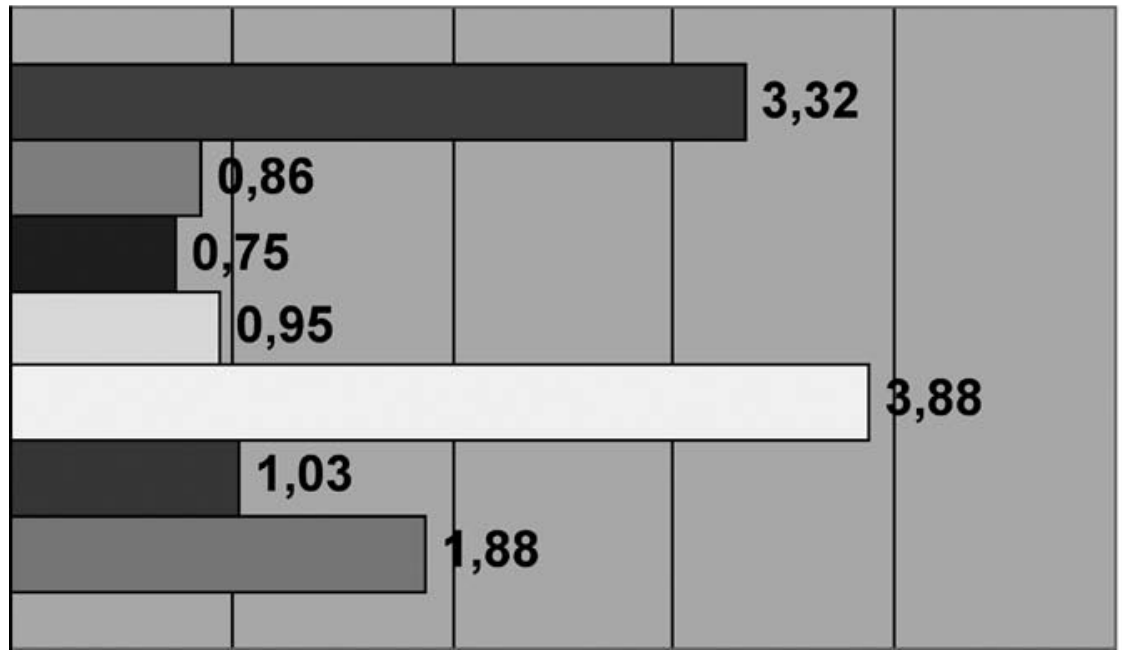

$\square$ higiene personal

sueño/vitalidad

emociones

rel. personales

limitaciones

actividades diarias

estado de salud

FIGURA 3. Variaciones de la componentes del KHQ.

3.- Si se ha sentido mejor ìha dejado de tomar alguna vez la medicación? Se observaron diferencias estadísticamente significativas (Z -6.557; $\mathrm{p}<0.001)$.

4.- Si en alguna ocasión se ha sentido mal al tomar la medicación ¿̇deja de tomarla? Se observaron diferencias entre la visita 4 y la final (Z -6.708; $p<0.001)$.

- Cumplimiento percibido por el paciente (EVA) (Figura 5)

EVA para la toma de tolterodina. Se observaron diferencias estadísticamente significativas entre la puntuación total media a las 4 semanas y la puntuación total media final (d 19.57; IC 95\%; 11.23$24.90 ;$ † $7.382 ; \mathrm{p}<0.001)$.

\section{Tolerancia y efectos adversos:}

Los efectos adversos achacables a tolterodina extended-release han sido: sequedad de boca $17,64 \%$, dispepsia $13,72 \%$ y sequedad lacrimal

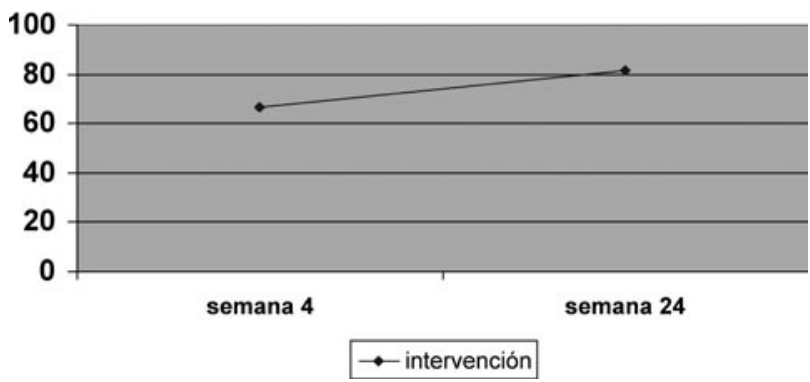

FIGURA 4. Evolución recuento de comprimidos de tolterodina.
$3,92 \%$. En ningún caso se ha producido abandonos por intolerancia o acontecimientos adversos.

\section{DISCUSIÓN}

El perfil sociodemográfico de los pacientes de este estudio se asemeja al perfil de los estudios de amplia base poblacional realizados en nuestro país (Rebollo y cols. 2003) 1, así como los llevados a cabo en Atención Primaria $(11,18)$.

Uno de los principales escollos en la realización del estudio ha sido el alto porcentaje de pacientes que han rechazado el estudio (el $34,78 \%$ ), una vez realizada la batería de cuestionarios en la visita inicial, debido probablemente a las fuertes connotaciones sexuales de algunos ítems del GRISS que todavía suponen un obstáculo cultural en la Atención Primaria. No sabemos la influencia que ha podido tener este rechazo como sesgo de selección, pero creemos que no invalida los objetivos del estudio aunque limite la aplicación de sus resultados.

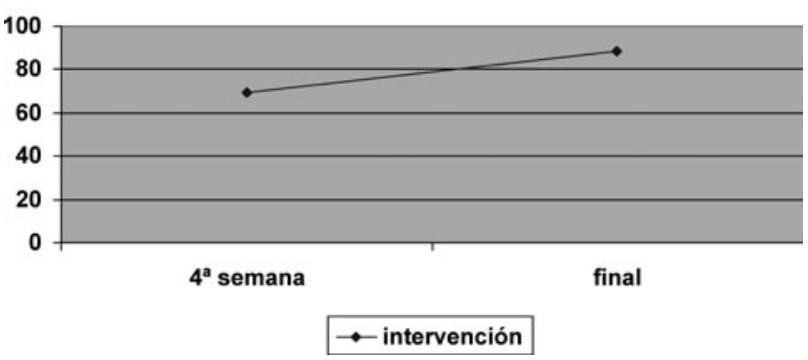

FIGURA 5. Evolución en la Escala Visual Analógica (EVA). 
En cuanto a las variaciones en el diario miccional, 6 de cada 10 pacientes han vuelto a situación previa normal, entendida como menos de 8 micciones diarias y/o menos de 2 por la noche, lo que viene a corroborar la eficacia conjunta de medidas de rehabilitación de suelo pélvico y terapia farmacológica $(18,24)$.

Los resultados en cuanto a mejora de la autopercepción de su estado de salud son muy esperanzadores, en el sentido de que su problema de incontinencia está influyendo negativamente en su percepción de salud, y en la medida en que intervengamos sobre la incontinencia de estos pacientes mejoraremos su "estado de salud". Estos datos se corroboran con los resultados del test $O A B-q$, específico de síntomas vesicales, y con los del $K H Q$ calidad de vida en incontinencia urinaria, en donde se ha producido una mejoría de la "misma intensidad" que la mejoría general en el estado de salud, no siendo esto atribuido a ningún otro factor que haya intervenido en estos pacientes a lo largo del estudio. Pese a que parte de esta mejoría puede deberse al importante efecto placebo, observado en múltiples ensayos clínicos, merece la pena tratar la incontinencia en Atención Primaria por lo beneficios específicos, estimados como el doble de la intervención respecto a placebo en dichos ensayos clínicos, y globales que comporta (20).

También se ha producido una mejora significativa en la percepción de calidad de vida de todas las componentes del KHQ en todos los pacientes estudiados y, en el caso de la función sexual, la mejora se ha focalizado en los aspectos relacionados con los síntomas de incontinencia. Esta mejoría relativa en calidad de vida tiene que ver con la importancia que tiene la sexualidad en la autoestima y en la autopercepción de salud del propio paciente (Cabello 2004) (21) y con el tratamiento farmacológico y rehabilitador.

En cuanto al número de abandonos por efectos secundarios ha sido similar a los publicados en los ensayos clínicos $y$, en general, tolterodina extended-release ha sido bien tolerada $(22,23)$.

En consonancia con lo anterior, el cumplimiento terapéutico ha mejorado por la eficacia de la intervención y por la escasa presentación de efectos adversos del tratamiento (24), su buena tolerancia y la dosificación una vez al día, puesto que estos son unos de los motivos claves en el cumplimiento terapéutico (25). No obstante aún queda mucho por mejorar en este aspecto puesto que hemos tenido que insistir en que la eficacia de tolterodina es máxima a partir de la $4^{a}$ semana de tratamiento (26), y estar muy pendientes de los controles de cumplimiento terapéutico para conseguir que la mayoría tomasen re- gularmente su medicación debido, fundamentalmente, a la variación de la intensidad de la incontinencia urinaria a lo largo del estudio y a las "necesidades sociales" de los pacientes, en el sentido de tomar la medicación en función de su programa de viajes, reuniones y otros eventos sociales.

\section{CONCLUSIONES}

A raíz de esta experiencia, recomendamos el uso sistemático en consulta de cuestionarios de cribaje para la incontinencia, y concretamente del ICIQ-SF (International Consultantion on Incontinente Questionnaire-Short Form) (7), validado en español por Monserrat Espuña y colaboradores en el 2004 que contempla la respuesta múltiple a 6 preguntas y cuyas cualidades psicométricas valoran la frecuencia, cantidad y afectación de la incontinencia. Entendemos que aunque en nuestro estudio hayamos usado el OAB-q con bastante utilidad, el ICIQ-SF es más simple y fácil de usar que el $O A B-q$, y además está avalado por el Comité Científico Internacional (Paris 2002) por lo que probablemente sea el cuestionario que más se vaya a utilizar en los estudios sobre incontinencia urinaria.

Cada vez más, como recomienda el informe de 2002 del Comité Científico Internacional, es más importante indagar en el impacto de la incontinencia en la sexualidad, aunque dicho Comité no sugiere ningún método específico. Nosotros hemos usado el cuestionario GRISS porque es un instrumento muy recomendado por los sexólogos para valorar la satisfacción sexual en la población general; sin embargo entendemos que no es la herramienta más adecuada para valorar específicamente el impacto en la sexualidad de pacientes con incontinencia urinaria puesto que únicamente han habido diferencias significativas en 2 de los 31 ítems de que consta el cuestionario, concretamente en los que hace referencia al rechazo a tener relaciones sexuales con su pareja y al escape de orina durante la relación sexual. Además, como hemos visto, la inclusión de este cuestionario ha tenido un efecto negativo en el reclutamiento de pacientes.

Finalmente, destacar la efectividad del tratamiento combinado de tolterodina extended-release y rehabilitación del suelo pélvico que, unidos a su tolerabilidad y fácil uso, los hace muy apropiados para el tratamiento integral de la incontinencia urinaria en Atención Primaria.

\section{AGRADECIMIENTOS}

A nuestro querido urólogo Alberto Murcia, por su insistencia en que mejoremos la atención a los incontinentes, a Pepe Lozano, amigo infatigable, 
a Francisco Molina por impulsar nuestro proyecto en el SMS, y a todo el equipo de Jose Luis Galiano de Pfizer por su apoyo.

\section{BIBLIOGRAFÍA Y LECTURAS RECOMENDADAS (*lectura de interés $y$ ** lectura fundamental)}

**1. REBOLLO, P.; ESPUÑA, M.: "Situación actual de del diagnóstico y tratamiento de la incontinencia de orina en España”. Arch. Esp. Urol., 56: 755, 2003.

2. BENTKOVER, J.D.; CHAPPLE, C.; COREY, R. y cols.: "Adapting a US cost-offset economic model for overactive bladder for the European marketplace". Val Health, 23: 78, 2003.

3. VERDEJO BRAVO, C.: "Incontinencia urinaria: un problema frecuente del paciente mayor". Ann. Med. Interna, Madrid, 17: 19, 2000.

*4. HANNESTAD, Y.S.; RORTVEIT, G.; SANDVIK, H. y cols.: "A community-based epidemiological survey of female urinary incontinence: The Norwegian EPINCONT study". J. Clin. Epidemiol., 53: 1150, 2000.

*5. BROWN, J.; McGHAN, W.; CHOKROVERTY, S.: "Comorbidities Associated With Overactive Bladder". Am. J. of Managed Care, 6: 574, 2000.

6. FERNÁNDEZ LÓPEZ, J.A.: "Estudios de calidad en Atención Primaria". Madrid, Nilo Industria Gráfica SA., 1998.

7. ABRAMS, P. y cols.: "2nd International Consultation on incontinente". Paris, Health Publication Ltd., 2002.

8. ABRAMS, P.; KELLEHER, C.J. y cols.: "Overactive bladder significantly affects quality of life". Am. J. of Managed Care, 6: 580, 2000.

*9. MARTÍNEZ AURA, F.; FOUZ LÓPEZ, P. y cols.: "Incontinencia urinaria: una visión desde Atención Primaria". MEDIFAM, 11: 55, 2001.

10. CASTRO DÍAZ, D.; POSTIUS ROBERT, J.: "Urología en Atención Primaria". Pulso Ediciones Barcelona, 2001.

11. GARCÍA-GIRALDA, L.; GUIRAO, L.; CASAS, I. y cols.: "Calidad de vida relacionada con la salud en pacientes con incontinencia urinaria en Atención Primaria". BERM, 25: 61, 2004.

12. FORTUNA, M.: "Tratamiento rehabilitador en incontinencia urinaria". Barcelona Soluciones 03, 2003.

13. NOE, L.; BECKER, R.; WILLIAMSON, T. y cols.: "A pharmacoeconomic model comparing two long-acting treatments for overactive bladder". JMCP, 5: 343, 2002.

14. KELLEHER, C.; KREDER, K.; PLEIL, A. y cols.: "Long-term health-related quality of life of patiens receiving extended-release tolterodine for overactive bladder". Am. J. of Managed Care, 8: 616, 2002.

*15. VAN KERREBROECK, P.H.; KREDER, K.; JONAS, U. y cols.: "Tolterodina una vez al día: eficacia y tolerabilidad superiores en el tratamiento de la vejiga hiperactiva (Tolterodine study group)". Urology, 3: 414, 2001.

16. THE-WEI, H.; WAGNER, T.: "Economic considerations in overactive bladder". Am. J. of Managed Care, 6: 591, 2000.

17. SACKETT, D.: "Compliance with therapeutics regimens". The Johns Hopins University Press, Baltimore, 1976.

18. GRUPO CORDOBÉS PARA EL ESTUDIO DE LA INCONTINENCIA URINARIA.: "Implantación de un programa en incontinencia urinaria en un centro de salud". Aten. Primaria, 31: 446, 2003.

19. FORÉS, M.M.; HORTELANO, M.A.; VIÑAS, R.: "Guía de Diagnóstico diferencial de incontinencia urinaria". Barcelona Soluciones 03, 2003.

**20. McGHAN, W.: "Cost effectiveness and quality of life considerations in the treatment of patiens with overactive bladder". Am. J. of Managed Care, 7: 62, 2001.

**21. CABELLO, F.: "Disfunción Eréctil: un abordaje integral". Madrid Psimática, 2004.

*22. CHANCELLOR, M.; FREEDMAN, S. y cols.: "Tolterodina, un tratamiento eficaz y bien tolerado de la incontinencia de urgencia y otros síntomas de vejiga hiperactiva". Clin. Drug Invest., 19: 83, 2000.

23. KREDER, K.; MAYNE, C.; JONAS, U.: "Longterm safety, tolerability and efficacy of extendedrelease Ttolterodine in the treatment of overactive bladder". European Urology, 41: 588, 2002.

24. VAN KERREBROECK, P.H.; KREDER, K.; JONAS, U. y cols.: "Tolterodina una vez al día: eficacia y tolerabilidad superiores en el tratamiento de la vejiga hiperactiva (Tolterodine study group)". Urology, 3: 414, 2001.

**25. MARQUEZ, E.; GIL, V.: "Variables que influyen en el incumplimiento del tratamiento farmacológico. Cumplimiento terapéutico en la HTA". Actualizaciones, 2: 3, 2005.

26. MIAMI, P.; SEIDMAN, L.; LAMA, D.: “A multicenter, prospective, open-label study of tolterodine extended-release $4 \mathrm{mg}$ for overactive bladder: the speed of onset of therapeutic assessment trial (STAT)". Clin. Ther., 24: 616, 2002.

*27. ESPUÑA, M.; REBOLLO, P.; PUIG, M.: "Validación de la versión española del International Consultation on Incontinente Questionnaire-Short Form. Un cuestionario para evaluar la incontinencia urinaria”. Med. Clin. 122:288, 2004. 